military in operational functions, and their spokesmen have taken part in broadcast interviews - but purely at a factual operational level.

The major issues still under review include: (1) Who is telling me? and (2) Can I trust them? Authorities must face the "fright factors" and "media triggers" and be ready in advance. But, there is a danger in having off-the-shelf, preprepared material that does not cover the precise details of a particular disruptive incident. Better to have generic material that can be adapted and pre-arranged conduits that can accept material at a few hours' notice.

Keywords: civil-military cooperation; command; communication; control; emergencies; government; information; preparedness;

public; responsibility; United Kingdom;

Prebosp Disast Med 2003;18(s1)s14.

\section{The Sagbata Project}

Dr. Flavio Del Ponte

Swiss Disaster Relief Unit, Geneva, Switzerland

The Dutch and Swiss government and NATO Civil Emergency Planning have initiated the Sagbala Project. The Project supports the need to enhance the protection of population in NATO and EAPC member countries against attacks with Weapons of Mass Destruction (WMD) with chemical, biological, radiological, and/or nuclear (CBRN) agents. The Sagbata Project will result in the creation of a set of tools (awareness raising/training support/decision support) to be used in WMD incidents for use by policy advisors to the political level. The initial subject matter area for these decision-support tools will be biological incidents. The tools will contain cause-andeffect relationships about consequence management in the early, uncertain phase of a WMD event.

The Sagbata tools are (named after the African god of smallpox) can be used by policy advisors to political decision-makers to prepare for a WMD event. The tool will help to provide insight in the consequences of the possible decisions taken during the various stages of a WMD disaster. This decision-support tool will be developed for awareness raising and training purposes. The tool eventually may be developed further into an operational, decision-support tool. Keywords: chemical, biological, radiological, and nuclear (CBRN) agents; decision-makers; decision-support; NATO; planning; policy; Sagbata Project; tool; training; weapons of mass destruction (WMD)

Prehosp Disast Med 2003;18(s1)s14.

\section{Development of an Operational Component in the International Committee of Military Medicine for Militaries to Support International Outbreak Alert and Response}

Major General Marcel Merlin, MD

Deputy Chair of the Scientific Council of the International

Committee of Military Medicine

Since 1921, more than 100 military health services have participated in the International Committee of Military Medicine (ICMM). The objectives of ICMM include: (1) Providing technical resources; (2) Preparing responses to disasters; (3) Promoting the implementation of a network of experts; and (4) Participating in training in the field of
International Humanitarian Law. Currently, an agreement of cooperation exists between the ICMM and the World Health Organization (WHO). A main goal approved by the last General Assembly of ICMM is the fostering of International Civil-Military Cooperation in responding to disasters. The disasters included can be the consequence of natural or technological events or conflicts. They include physical, radiological, chemical, and biological hazards of natural, accidental, or deliberate origin. There is a very high impact associated with the use of biological weapons and the fact that terrorism must resort to attacks using such weapons to activate fear in the target population. In such a context, the use of biological weapons must be regarded as a true threat. Currently, the WHO is implementing a program to alert possible victims and develop responses to emerging diseases, including epidemics of deliberate origin. In Washington during September 2004, a draft of a standardized agreement will be proposed to the General Assembly of the ICMM, which, if approved, could be used by the WHO to negotiate with the government of each member state of the ICMM, for the participation of its Military Health Service in responding along with civilian responders to public health emergencies in the international arena.

Keywords: alert; biological weapons; civilian Military cooperation; international assistance; International Committee of Military

Medicine (ICMM); public health emergencies; response, inter-

national; World Health Organization

Prehosp Disast Med 2003:18:s(1)s14.

\section{Civil Military Cooperation through the Laboratory Response Network}

Stephen A. Morse, MSPH, PbD

Bioterrorism Preparedness and Response Program, Centers for Disease Control and Prevention, Atlanta, Georgia USA

The Laboratory Response Network (LRN) is a national system designed to link state and local public health laboratories with other advanced-capacity, clinical, military, veterinary, agricultural, water, and food-testing laboratories including those at the federal level. The LRN is a critical component of CDC's public health mission, enhancing U.S. readiness to detect and respond to bioterrorism incidents. Operational in August, the LRN has the ability to detect and respond to agents that are released by a bioterrorist as well as those that occur naturally. There currently are 120 LRN reference laboratories in the U.S. and Canada, and further expansion is planned. In addition to bio-threat agents, the LRN is expanding to include the ability to detect chemical agents. The CDC's role through the LRN is to support the public health infrastructure, which is defined by pubic health laboratory work. Standardized laboratory protocols are available for each agent through a secure web site. In addition, CDC is mandated to produce, validate, package, and ship reagents for most of these screening and confirmatory procedures. Training and a proficiency-testing program also are provided by the CDC. The LRN played a critical and successful role in the U.S. response to the bioterrorism-related anthrax incidents of 2001. The LRN laboratories, including those of the military, tested $>125,000$ clinical specimens and environmental samples involving approximately 
1 million assays. The LRN has a dual function, since the introduction of advanced technology and training into the nation's public health laboratories has facilitated the response to emerging disease threats such as SARS.

Keywords: anthrax; bioterrorism; bio-threat; Center for Disease Control and Prevention (CDC); civil-military cooperation; laboratories; Laboratory Response Network (LRN); public health; system; testing

Prehosp Disast Med 2003;18(s1)s15.

\section{New Concept for Multi-disciplinary Crisis Management \\ Brigadier Dag HjeIIe, MD \\ Norwegian Armed Forces Medical Center}

After the end of the cold war era, new conflicts and challenges have been brought upon us. The threats from international terrorism are among many challenges to our preparedness systems. Others types of crisis, as the SARS epidemic, also must be handled. All together, there is a large variation in the types of threats that range from civilian accidents to full-scale war. How do we cope with such threats in a trustworthy way and within reasonable budgets?

Many of these threats have medical implications. The Norwegian Armed Farces Medical Services together with a large civilian medical institution, Ullevaal University Hospital, has constructed a new concept in which medical resources, both clinical competence and laboratory services are put together in a way that they can support civilian medical operative needs, military medical operative needs, and at the same time, support decision makers in both the civilian and military chains of command. The focus has been on operative usefulness, to facilitate flexible and scalable reaction abilities, and also to enable pro-active responses. In principle, each unit works along three lines, one operative civilian medical line, one operative military med cal line, and one linked to the military and civilian chains of command through a medical operations centre The main resources linked directly to the centre is medical microbiology / infectous deseases management (ablilty to isolate patients in an isolation centre at P3 level and a diagnostic laboratory at P3/ P4 level), toxicology, psychiatry (crisis management, psychological trauma care), trauma care (surgery and emergency medicine), radiation medicine, and a military medical operations centre linked to the civilian emergency response system. The system also is linked to a medical intelligence cell and the medical preparedeness branch within the Armed Forces Medical Services.

Keywords: chains of command; civil-military cooperation; command and control; crisis management; emergency response system; Norwegian Armed Forces; terrorism; threats

Prehosp Disast Med 2003;18(s1)s15.
Civil-Military Cooperation in Responding to the Anthrax Attacks in 2001

Major Tony Littrell, MD, MPH

Chief, Field Operations \& Intelligence, USAMRIID (United

States Army Medical Research Institute of Infectious Diseases, Fort Detrick, Maryland, USA)

In October of 2001 at least four letters containing anthrax spores were mailed to various prominent figures in the United States government and media organizations. What followed is perhaps the largest bioterrorism investigation and public health response in U.S. history. Physicians and scientists from the United States Army Medical Research Institute of Infectious Diseases (USAMRIID) played an important role in responding to this public health emergency. This session will highlight the cooperation and lessons learned between civilian public health organizations and USAMRIID employees. We will also discuss what actions can be taken to improve cooperation and bioterrorism response plans in the future.

Keywords: anthrax; bioterrorism; civil-military cooperation; USAMRIID

Prehosp Disast Med 2003:18:s(1)s15.

\section{Wednesday, 03 September Chemical/Biological Threat}

\author{
Co-operation in the European Union on \\ Preparedness and Response to Biological and \\ Chemical Agent Attacks (Health Security) \\ Germain Thinus \\ Health Security, Task Force European Commission
}

The actions of the European Union (EU) relative to terrorism has been outlined in three communications issued by the European Commission: (1) November 2001, (2) June 2002, and (3) June 2003, that deal in detail with the health aspects of this action, (communication COM (2003) 320 final). Moreover, a joint programme of action for chemical, biological, and radio-nuclear threats was agreed by the EU Council of Ministers and the Commission on 20 December 2002, as requested by the European Council (heads of State and Government of the $\mathrm{EU}$ ) in Ghent, Belgium in October 2001. This programme sets out the key objectives of action on CBRN threats across the policies and sectors of activities of the EU, and lists the legal instruments that can be used to counter such threats.

In the area of health, guidelines agreed upon at the Health Council of the EU on 15 November 2001, led to the drawing-up of the 25 -action programme on health security, currently being implemented in close collaboration between the Commission and the EU Member States, which aims to contribute towards:

1. EU-wide capability for the timely detection and for the identification of biological and chemical agents in laboratories;

2. Rapid and reliable determination and diagnosis of human disease cases;

3. Availability of medicines; 